\title{
Size stabilization of surface-supported liquid aerosols using tapered optical fiber coupling
}

\author{
Yasin Karadag, ${ }^{1}$ Alexandr Jonášs, ${ }^{1,4}$ Ibrahim Kucukkara, ${ }^{1,3}$ and Alper Kiraz ${ }^{1,2,5}$ \\ ${ }^{1}$ Department of Physics, Koç University, Rumelifeneri Yolu, Sariyer, Istanbul 34450, Turkey \\ ${ }^{2}$ Koç University TÜPRAS Energy Center (KÜTEM), Koç University, Rumelifeneri Yolu, Sariyer, Istanbul 34450, Turkey \\ ${ }^{3}$ Current address: Department of Physics, Mersin University, Mezitli, Mersin 33343, Turkey \\ ${ }^{4} e$-mail: ajonas@ku.edu.tr \\ ${ }^{5} e$-mail: akiraz@ku.edu.tr
}

Received January 11, 2013; accepted January 24, 2013;

posted February 5, 2013 (Doc. ID 183229); published February 28, 2013

\begin{abstract}
We demonstrate long-term size stabilization of surface-supported liquid aerosols of salt-water. Single tapered optical fibers were used to couple the light from independent heating and probe lasers into individual microdroplets that were kept on a superhydrophobic surface in a high-humidity chamber. Size stabilization of microdroplets resulted from competition between resonant absorption of the infrared heating laser by a microdroplet whispering gallery mode and water condensation in the sample chamber. Microdroplet size was continuously monitored using the tunable red probe laser. Thanks to the narrow linewidth of the heating laser, stabilization of the $110 \mu \mathrm{m}$ radius of a microdroplet with a precision down to $0.54 \mathrm{~nm}$ was achieved for a period of $410 \mathrm{~s}$. @ 2013 Optical Society of America

OCIS codes: $\quad 140.3945,060.2340,010.1110,300.6320$.
\end{abstract}

Liquid aerosol research is important for a large variety of fields including atmospheric chemistry and physics, combustion science, plasma physics, and health science $[1,2]$. Such studies require experimental tools for simultaneous manipulation and high-resolution characterization of individual liquid aerosol particles. This is a challenging task due to the airborne nature of aerosols, which makes them difficult to localize and renders their chemical and physical properties (e.g., composition, size and mixing state) very sensitive to the composition and temperature of the surrounding gas. Localization of liquid aerosols has been, to date, successfully achieved using various approaches including optical levitation [3], electrodynamic levitation [4], acoustic levitation [5], optical tweezers [6], and superhydrophobic (SH) surfaces [7]. In contrast, precise size stabilization is still an unresolved issue that limits prolonged studies of individual liquid aerosol particles. Currently, size stabilization of liquid aerosols is mostly accomplished by adding nonvolatile components (e.g., inorganic salt or glycerol) into their composition and keeping them in chambers with fixed environmental conditions. This approach, however, is insufficient for high-resolution aerosol characterization due to difficulties in precise control of the environmental conditions leading to size instabilities of the liquid aerosol particles.

Recently, an alternative method for self-stabilization of the size of aqueous microdroplets based on heating with a free-space propagating laser beam has been demonstrated and successfully applied to microdroplets immobilized on a SH surface $[8,9]$ or by optical tweezers [10]. In these demonstrations, stable locking of the droplet size resulted from competition between droplet growth due to condensation in a high-humidity chamber and shrinkage due to the evaporation caused by resonant absorption of the heating laser light either directly by water $[9,10]$ or by a dissolved fluorescent dye [8]. The stability of the droplet size was characterized by monitoring the spectral positions of the droplet whispering gallery modes (WGMs) via fluorescence spectroscopy

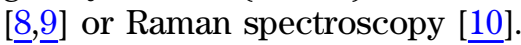

0146-9592/13/050793-03\$15.00/0
Here, we demonstrate long-term size stabilization of individual microdroplets standing on an SH surface and coupled to single tapered optical fibers, which guide simultaneously a tunable red laser for WGM probing [11] and an infrared (IR) heating laser. The use of a tapered optical fiber for coupling both lasers into the droplets increases significantly the coupling efficiency in comparison to the free-space arrangement adopted previously [12]. Moreover, droplet size stabilization and high-resolution WGM spectroscopy can be carried out concurrently, with almost no increase in the setup complexity. We note that self-stabilization of a solid microcavity coupled to a tapered fiber has been previously shown [13] using cavity expansion and refractive index change caused by laser heating as the underlying physical mechanisms.

The experimental setup and procedures used for size stabilization of surface-supported liquid microdroplets were similar to those described in [11]. SH surfaces were prepared by spin coating of hydrophobic silica nanoparticles suspended in isopropanol on cleaned glass substrates. Subsequently, individual microdroplets of an aqueous solution of $\mathrm{MgCl}_{2}$ (initial concentration $2 \mathrm{mM}$ ) with a typical radius $a=100-200 \mu \mathrm{m}$ were manually deposited on the SH surface with a glass microcapillary. A fiber taper manufactured from a single-mode optical fiber optimized for $633 \mathrm{~nm}$ by the heat-and-pull technique served to couple both probe and heating lasers into and out of the microdroplet $[11,12]$. An $X Y Z$ piezo stage was used to position the taper within the proximity of the droplet to allow light coupling. The microdroplet deposited on the SH surface was kept in a sealed chamber with its relative humidity fixed at $84 \%$ by a saturated aqueous solution of $\mathrm{KCl}$.

Tunable probe lasers $\left(\lambda_{P}\right.$ around 635.5 or $638.0 \mathrm{~nm}$, linewidth $<1 \mathrm{MHz}$ ) and a fixed wavelength heating laser $\left(\lambda_{H}=1064 \mathrm{~nm}\right.$, linewidth $\left.<17 \mathrm{MHz}\right)$ were combined in the same tapered fiber using a fused fiber coupler. In order to minimize the absorption of the tunable probe laser light in the droplet liquid, we performed the measurements in the visible part of the spectrum where water is virtually nonabsorbing. In contrast, the heating laser wavelength 
was selected to be in the IR region where water absorption is more than two orders of magnitude larger than that at visible wavelengths.

Laser-induced heating of droplets depends strongly on the droplet radius $a$. When $a$ is in resonance with the heating laser wavelength, light absorption and associated heating can increase by several orders of magnitude. As shown by Karadag et al. [9] , the size of a growing droplet that is initially out of resonance with heating laser WGMs can be stabilized at a certain size parameter $\alpha_{S}=2 \pi a_{S} / \lambda_{H}$, at which the water evaporation and condensation precisely balance each other; this $\alpha_{S}$ is slightly blue-detuned with respect to a WGM of the heating laser. Upon reaching $\alpha_{S}$, further increase of size above $a_{S}$ tunes the droplet closer to the absorption resonance. This in turn causes increased heating, which drives the droplet back to $\alpha_{S}$. Similarly, decrease of size with respect to $a_{S}$ leads to a stronger condensation since the heating power decreases further from the resonance. Again, the droplet is driven back to $\alpha_{S}$. This mechanism then leads to selflocking of the droplet size.

Figure 1(a) shows a two-dimensional (2D) plot of consecutive WGM spectra recorded from a microdroplet exhibiting size locking. Laser powers used in this experiment were measured at the output of the fiber taper as $16.5 \mu \mathrm{W}$ and $0.35 \mathrm{~mW}$ for the probe and heating lasers, respectively. The average acquisition time of an individual spectrum (a single horizontal line in the image) was $2.06 \mathrm{~s}$. Prior to the spectrum acquisition, the microdroplet was prepared at $73 \%$ ambient relative humidity and then placed in a closed sample chamber with humidity fixed at $84 \%$. Hence, upon chamber loading, microdroplet growth due to gradual condensation was observed. Results shown in Fig. 1 were recorded immediately after the chamber loading, before the microdroplet reached its equilibrium size. For times $t<410 \mathrm{~s}$, both the probe and the heating lasers were coupled to the tapered fiber. During this period, the spectral positions of the WGMs remained virtually constant with only a small red shift $\Delta \lambda=3.1 \mathrm{pm}$ over the total acquisition time. This slow spectral shift can be attributed to the gradual increase

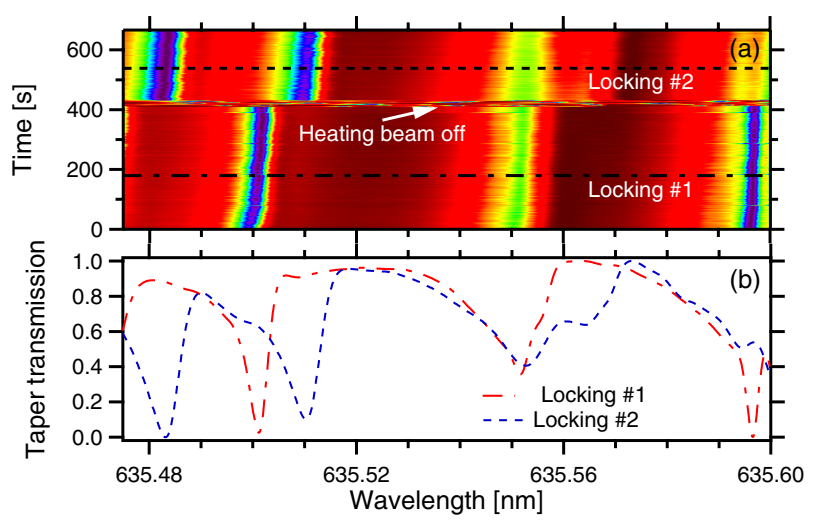

Fig. 1. (Color online) (a) 2D plot of consecutive WGM spectra of a droplet stabilized by IR laser heating. At time $t=410 \mathrm{~s}$, the heating laser was blocked during 10 spectral acquisitions and then unblocked again, resulting in a change of the stabilized droplet size. (b) Details of the droplet WGM spectra along the dash-dotted and dashed lines shown in (a). Mean droplet radius was $110 \mu \mathrm{m}$. of the chamber relative humidity toward its saturation value. With higher humidity, the condensation rate increases and, thus, a new equilibrium between evaporation and condensation is established at a slightly longer wavelength closer to the heating beam resonance. $\Delta \lambda$ can be converted into the residual drift of the droplet radius $\Delta a$ as $\Delta a=\Delta \lambda\left(a / \lambda_{P}\right)$ [11]. For $a=110 \mu \mathrm{m}$ and $\lambda_{P}=$ $635.5 \mathrm{~nm}, \Delta a=0.54 \mathrm{~nm}$. For times $410 \mathrm{~s}<t<430 \mathrm{~s}$, the heating laser was blocked, which resulted in a large spectral drift of the droplet WGMs toward the red end of the spectrum due to condensation in the chamber. Subsequently, the heating laser was unblocked again, starting at time $t=430 \mathrm{~s}$ until the final acquisition time $t=635 \mathrm{~s}$. During this period, the spectral positions of the WGMs were again almost constant, suggesting size stabilization of the microdroplet with a similar rate of the residual drift of the droplet size as in the first locking period. We note that the spectral positions of the WGMs during the first $(t=\langle 0-410\rangle \mathrm{s})$ and second $(t=\langle 430-635\rangle \mathrm{s})$ size-stabilized periods are not the same [see Fig. 1(b)]. This indicates locking of the microdroplet to different absorption resonances of the heating laser, which correspond to different droplet sizes.

In order to study the dynamics of droplet selfstabilization after a sudden change in environmental conditions, we carried out experiments in which we periodically modulated the heating laser power and monitored the droplet response. Figures $\underline{2}$ and $\underline{3}$ provide an illustration of the droplet size-locking dynamics. In Fig. 2(a), a spectrum of relatively low- $Q$ WGMs $(Q$ up to $\approx 4.0 \times 10^{5}$ ) in a size-stabilized droplet is shown. After acquiring this spectrum, the probe laser was tuned to the droplet WGM wavelength indicated by an arrow in Fig. 2(a). Subsequently, the heating laser was repeatedly blocked for $100 \mathrm{~ms}$ and then unblocked again and the taper transmission was simultaneously recorded at the fixed WGM wavelength. The time trace of the recorded taper transmission is shown in Fig. 2(b). In the time interval between 0 and $1 \mathrm{~s}$, the droplet size is locked and the taper transmission remains constant at its

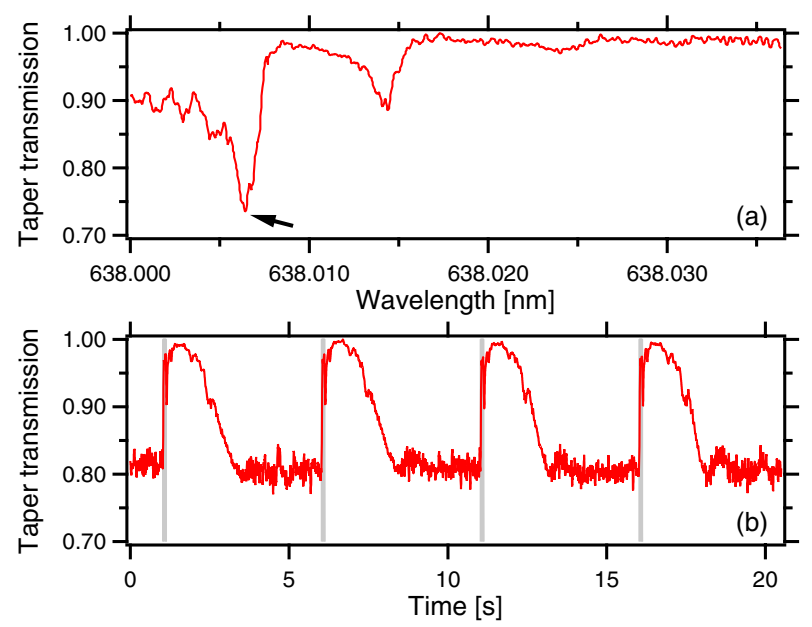

Fig. 2. (Color online) (a) High-resolution WGM spectrum of size-locked microdroplet with $110 \mu \mathrm{m}$ radius. (b) Time trace of the taper transmission at a fixed wavelength of the probe laser denoted by the arrow in (a). Gray stripes indicate time intervals during which the heating laser was blocked. 

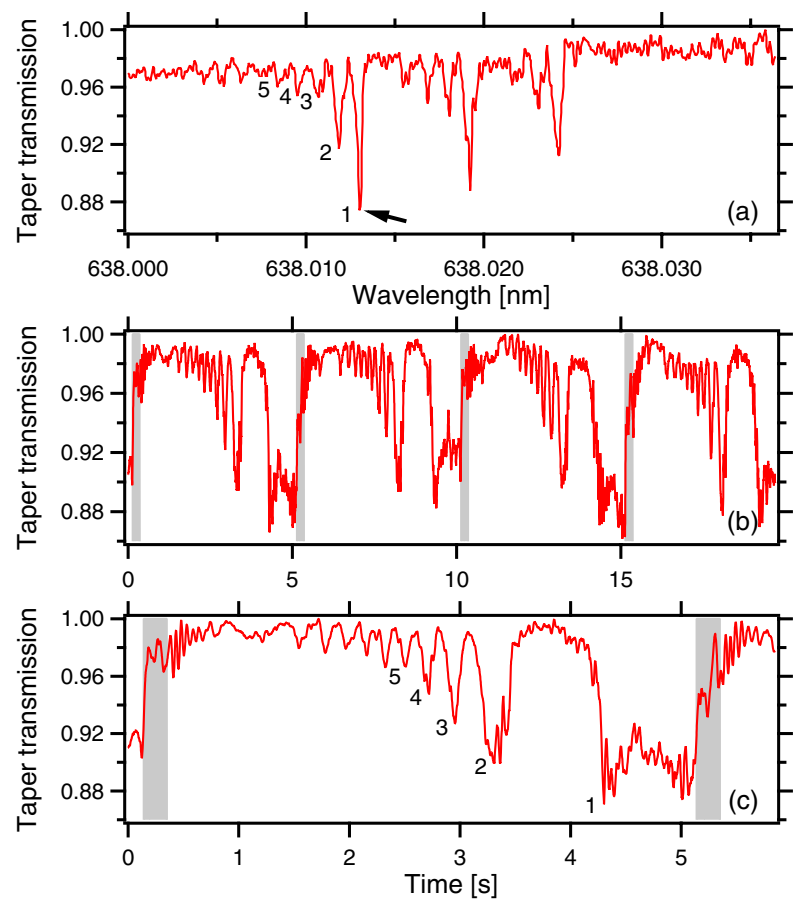

Fig. 3. (Color online) (a) High-resolution WGM spectrum of a size-locked microdroplet with $160 \mu$ m radius. (b) Time trace of the taper transmission at a fixed wavelength of the probe laser denoted by the arrow in (a). Gray stripes indicate time intervals during which the heating laser was blocked. (c) Detail of the time trace shown in (b).

minimum value corresponding to the WGM resonance. At time $t=1 \mathrm{~s}$, brief blocking of the heating laser leads to a fast increase in the droplet size. Since the probe wavelength is fixed, such an increase in the droplet size corresponds to an increase in the droplet size parameter. This is equivalent to a shift to the blue end of the spectrum of Fig. 2(a) that was acquired with a constant droplet size and changing wavelength. Thus, taper transmission quickly increases. After unblocking the heating laser, the droplet starts evaporating again and the droplet size parameter eventually decreases back toward its resonant value. This results in a gradual decrease of the taper transmission with an intensity profile that follows the spectral features of the blue shoulder of the selected WGM resonance. Figure 3 shows a similar dynamic response recorded for high- $\bar{Q}$ WGMs $\left(Q\right.$ up to $\left.\approx 2.1 \times 10^{6}\right)$ with a largely lifted degeneracy of the different azimuthal modes [see Fig. 3(a)]. In this case, the temporal profile of the taper transmission is rather complex, reflecting again the shape of the spectrum in the blue shoulder of the selected WGM resonance. Indeed, the depths of individual spectral dips observed in the wavelength scan and the time trace [compare corresponding dip numbers in Figs. 3(a) and 3(c)] are in a good agreement. As shown in Figs. 2(b) and 3(b), upon repeated blocking of the heating laser, the shape of the temporal response remains almost constant. This indicates that the whole process is reversible.

We have demonstrated long-term self-stabilization of the size of salt-water aerosols standing on an SH surface and coupled to a tapered optical fiber guiding independent probe and heating lasers. The size self-stabilization resulted from competition between resonant heating of the droplets by the IR heating laser and water condensation in an ambient environment with controlled relative humidity. Thanks to the narrow linewidth of the heating laser, stabilization of the microdroplet radius with a precision down to $0.54 \mathrm{~nm}$ was achieved. This technique can be useful for ultrahigh-resolution characterization of liquid aerosols and applications of liquid optical microcavities that require size-stabilization, such as frequency combs [14].

This work was partially supported by TÜBİTAK (Grant Nos. 109T734 and 111T059), EC Marie Curie IEF (Contract No. PIEF-GA-2009-252579; A.J.), and FABED Young Investigator Research Award (A.K.).

\section{References}

1. J. P. Reid and L. Mitchem, Annu. Rev. Phys. Chem. 57, 245 (2006).

2. D. McGloin, D. R. Burnham, M. D. Summers, D. Rudd, N Dewar, and S. Anand, Faraday Discuss. 137, 335 (2008).

3. A. Ashkin and J. M. Dziedzic, Science 187, 1073 (1975).

4. S. Arnold and L. M. Folan, Rev. Sci. Instrum. 57, 2250 (1986).

5. H. Azzouz, L. Alkhafadiji, S. Balslev, J. Johansson, N. A Mortensen, S. Nilsson, and A. Kristensen, Opt. Express 14, 4374 (2006).

6. R. J. Hopkins, L. Mitchem, A. D. Ward, and J. P. Reid, Phys. Chem. Chem. Phys. 6, 4924 (2004).

7. A. Kiraz, A. Kurt, M. A. Dündar, and A. L. Demirel, Appl. Phys. Lett. 89, 081118 (2006).

8. A. Kiraz, A. Kurt, M. A. Dündar, M. Y. Yüce, and A. L. Demirel, J. Opt. Soc. Am. B 24, 1824 (2007).

9. Y. Karadag, M. Mestre, and A. Kiraz, Phys. Chem. Chem. Phys. 11, 7145 (2009).

10. M. Guillon, R. E. H. Miles, J. P. Reid, and D. McGloin, New J. Phys. 11, 103041 (2009).

11. A. Jonáš, Y. Karadag, M. Mestre, and A. Kiraz, J. Opt. Soc. Am. B 29, 3240 (2012).

12. J. C. Knight, G. Cheung, F. Jacques, and T. A. Birks, Opt. Lett. 22, 1129 (1997)

13. T. Carmon, L. Yang, and K. J. Vahala, Opt. Express 12, 4742 (2004).

14. P. Del'Haye, A. Schliesser, O. Arcizet, T. Wilken, R. Holzwarth, and T. J. Kippenberg, Nature 450, 1214 (2007). 